\title{
A Systematic Review of Sudden Unexpected Death in Epilepsy (SUDEP) in Childhood
}

Omar Abdel-Mannan BMBCh ${ }^{\mathrm{a}}$, Henry Taylor MBBS ${ }^{\mathrm{b}}$, Elizabeth J. Donner MD ${ }^{\mathrm{c}}$, Alastair

G. Sutcliffe $P h D^{d}$

${ }^{a}$ Department of Neurology, Great Ormond Street Hospital for Children NHS Trust, London, United Kingdom.

${ }^{\mathrm{b}}$ Department of Paediatrics, Northwick Park Hospital, London North West Healthcare NHS Trust, London, United Kingdom.

${ }^{c}$ Department of Neurology, The Hospital for Sick Children, Toronto, Canada

${ }^{\mathrm{e}}$ Population, Policy and Practice Unit, UCL Great Ormond Street Institute of Child Health, London, United Kingdom.

Address correspondence to: Omar Abdel-Mannan, Department of Neurology, Great

Ormond Street Hospital for Children, London, Great Ormond Street, London, WC1N 3JH

[o.abdel-mannan@ucl.ac.uk], 07717747012

Funding Source: No funding was secured for this study.

Financial Disclosure: All authors have no financial relationships relevant to this article to disclose.

Conflict of Interest: All authors have no conflicts of interest to disclose.

Number of words (main text): 3054

Key words: Sudden Unexpected Death in Epilepsy, Sudden Unexplained Death in Epilepsy, SUDEP, Epidemiology, Epilepsy mortality 


\section{Abstract}

Background: Sudden Unexpected Death in Epilepsy (SUDEP) is a significant cause of death in childhood epilepsy, and causes considerable concern to patients and their families. Despite this, the condition remains poorly understood. This systematic review investigates the risk factors, pathophysiology and circumstances associated with childhood SUDEP. It aims to explore the etiology of SUDEP, and inform clinicians approaching SUDEP risk disclosure.

Methods: A structured electronic database search of Medline, Central, Embase, and ISI web of science was conducted. Studies were included if they described clinical details of one or more patients, aged 18 years of age and below, who died of SUDEP. Two reviewers independently reviewed each article for data extraction and quality assessment.

Results: Information on 108 cases of pediatric SUDEP was extracted from 22 included studies. These comprised five cohort studies, four retrospective case control studies, seven case series, and five case reports. Factors which appeared to be linked to pediatric SUDEP included those associated with severe epilepsy (early age of onset, high seizure frequency, intellectual impairment and developmental delay, multiple anti-epileptic drug therapy, and structural abnormalities). The majority of included studies were non-comparative and had significant risk of bias.

Conclusions: There is currently insufficient evidence to determine the etiology of pediatric SUDEP. Current best practice to prevent pediatric SUDEP is to optimise the management of epilepsy. A national SUDEP registry would provide invaluable high-quality data and insights into modifiable risk factors, genetic predispositions, and novel prevention strategies. 


\section{Introduction}

In addition to increased morbidity and rates of hospitalisation, people with epilepsy are 2 to 3 times more likely to die early than the general population. ${ }^{1}$ Both adults and children with epilepsy may die from a number of causes including; complication of a seizure such as aspiration of stomach contents, suffocation, injury, drowning, status epilepticus, a related underlying condition (e.g. brain tumour), suicide, or Sudden Unexpected Death in Epilepsy (SUDEP). The most widely used definition of SUDEP is "the sudden, unexpected, witnessed or unwitnessed, non-traumatic and non-drowning death in patients with epilepsy, with or without evidence for a seizure, and excluding documented status epilepticus, in which postmortem examination does not reveal a toxicological or anatomical cause for death". ${ }^{2}$ In the most recently proposed system, SUDEP is sub-classified into definite, probable, possible SUDEP. ${ }^{3}$ (Figure 1)

\subsection{What do we know about SUDEP incidence?}

In adults, reported SUDEP rates vary greatly between studies depending on the studied cohort and range from as high as 6:1000 person-years in intractable epilepsy surgery cohorts ${ }^{4}$ to as low as $0.35 / 1,000$ person-years in a population-based study. ${ }^{5}$ This reported variation in adult SUDEP incidence is also mirrored in the pediatric population. Although the literature indicates the rate of SUDEP in children is lower than in adulthood, a recent systematic review as part of the AAN Practice Guideline on SUDEP found that the SUDEP risk in children with epilepsy is $0.22 / 1,000$ patient-years $(95 \%$ CI $0.16-0.31){ }^{6}$ 


\subsection{What have we learnt from the adult literature?}

There have been few controlled studies to date looking at childhood SUDEP risk factors; the majority of larger studies have included mostly adults. The studies are not without limitations, including: small numbers of SUDEP cases, inappropriate choice of controls, retrospective design, misclassification of SUDEP cases, lack of uniformity in definitions, and use of antiepileptic drug (AED) prescriptions to ascertain cases. ${ }^{7-9}$ The Task Force on Epidemiology of the International League Against Epilepsy (ILAE) pooled data from four major case-control studies of SUDEP. ${ }^{10-13}$ and showed that the risk of SUDEP was 1.4 times higher in male patients than in female patients. However, the recent AAN practice guideline systematic review determined that male sex was not a significant risk factor and that the presence and frequency of generalised tonic-clonic seizures (GTCS) was the major risk factor associated with SUDEP. ${ }^{6}$

\subsection{What are the pathophysiological mechanisms underlying SUDEP?}

There has been intense on-going study into possible mechanisms for SUDEP, and it remains unknown whether pediatric and adult SUDEP share a common mechanism. In general, the proposed mechanisms of SUDEP are cardiac, respiratory or central/autonomic dysregulation, all of which may occur independently or in combination. ${ }^{14}$ A proposed SUDEP mechanism, based on a retrospective review of 16 definite or probable SUDEP cases from 147 epilepsymonitoring units of cardiorespiratory arrests during video-EEG recordings suggests an early, centrally mediated alteration of both respiratory and cardiac functions occurs after GTCS. ${ }^{15}$

A better understanding of the risk factors, pathophysiology and circumstances associated with 
childhood SUDEP deaths would aid clinicians in several ways. First any modifiable risk factors could be targeted to reduce the incidence of SUDEP further. Second clinicians will be more able to approach SUDEP risk disclosure in an evidence-based manner with families when deemed appropriate. ${ }^{16}$ Relatives of both adult and child patients with SUDEP consistently indicate that they wish they had been informed that epilepsy can be fatal. ${ }^{17}$ Finally clues to the mechanism and etiology of SUDEP could be derived from the risk factors, prompting further research. Therefore, the aim of this paper is to systematically review the limited literature on childhood SUDEP, focusing on knowledge of risk factors of this important clinical entity.

\section{Methods}

This review was conducted according to the Preferred Reporting Items for Systematic Reviews and Meta-Analysis (PRISMA; Supplementary Information) guidelines.

\subsection{Search strategy and selection of studies}

A structured electronic database search of the Cochrane Central Register of Controlled Trials (CENTRAL), MEDLINE (1966 to February 2018) EMBASE, and ISI Web of Science (1945 to February 2018) was conducted with no language restriction (Supplementary Table 1). The reference list and ISI citations of all included studies were hand searched, as were the conference abstract issues of selected neurology journals for the year preceding our electronic search.

Studies were included if they described clinical details of one or more patients, aged 18 years of age and below, who died of SUDEP. Studies which described adult and pediatric cases were included if information on only the pediatric cases was available (either published or 
provided in correspondence with study authors). Studies were excluded if only combined adult and pediatric data were available.

Two authors [OA, HT] independently screened titles and abstracts of all studies identified in the literature search. Independent full text review of all potentially eligible studies was also conducted by two authors [OA, HT]. Any disagreements were resolved through discussion, or through consultation with a senior author [AS].

Duplicates were removed using EndNote software. Multiple reports of the same study were collated. Authors were contacted via e-mail if further data were required, or if there was no published subgroup analysis of pediatric cases.

\subsection{Data Extraction and Analysis}

Two authors [OA, HT] extracted study characteristics and outcome data from included studies using a standardised data collection form. Disagreements were resolved through discussion or consultation with senior author $[\mathrm{AS}]$. The following details were extracted: patient population, number of patients, age of patients, study design, duration and follow-up, outcome measures, study findings, author conclusions based on findings, strengths and limitations of the study.

Where studies included individual pediatric cases of SUDEP, we extracted the following information for each case: age at death, age at onset of epilepsy, epilepsy syndrome/diagnosis/etiology of epilepsy, types of seizures, frequency of seizures, circumstances at death, medication status prior to death, anti-epileptic drug levels at autopsy, co-morbidities, autopsy findings, cause of death (definite, probable, possible SUDEP), any other relevant information. 
Risk of bias assessment was conducted independently by two authors [HT, OA] using an assessment tool adapted from the effective public health practice project (Supplementary Information). Disagreements of a single point were resolved through discussion where possible, all others were reviewed by a senior author [AS].

\section{Results}

\subsection{Search findings}

A total of 590 possible titles were identified in the electronic database search and ten were identified through hand-searching citation lists (Figure 2). Following title and abstract review 497 papers were excluded on the basis that they did not include data on cases of SUDEP in children. Forty-five duplicate papers were identified and excluded, as were 12 papers for which the full text could not be obtained. During full text review eight papers were excluded as they did not meet inclusion criteria, and 17 were excluded as individual case information was not available and could not be provided by the study authors (Supplemental Table 3).

From the 22 included studies (Table 1) we were able to obtain individual information for 108 children who died of SUDEP (Figure 3). ${ }^{18-39}$ Five of the 22 included studies were cohort studies, four were retrospective case control studies, eight were case series, and five were case reports.

\subsection{Risk of bias}

Risk of bias was assessed for all included studies (Supplemental Table 2). There was good agreement between the two authors (complete agreement for $94 \%$ of measures, minor difference resolved by discussion for $6 \%$ of measures). 


\subsection{Case demographics}

There was a male predominance of SUDEP cases $(51 \%)$ over females $(38 \%)$. The mean age at seizure onset was 3.1 years (standard deviation (SD) 4.3 years, range 1 month-17 years). The average age at death was 10.2 years (SD 5.4 years, range 7 months to 18 years).

SUDEP cases were classified as definite (66 cases, 61\%), probable (26 cases, 24\%), and possible (16 cases, 15\%), as classified by the study authors according to definition criteria used by Nashef and Annegers. ${ }^{3,67}$ An autopsy +/- histological examination of the brain was performed in 74 cases.

\subsection{Seizure characteristics}

Types of seizure were reported for 60 patients. Fifty-three had generalised tonic-clonic seizures $(88 \%)$ and 20 had either simple or partial complex seizures $(33 \%)$. Seizure frequency was reported in only 12 patients, with an average of 16.3 seizures per month (SD 14.6, range 0.5 to 45$)$

\subsection{Epilepsy characteristics}

The etiology of epilepsy was reported for 81 patients, using variable terminology dependent on the year of publication. Twenty-seven children had symptomatic epilepsy, now subcategorized as either structural, metabolic or immune (33\%). Seventeen patients were described as having idiopathic epilepsy, now referred to as genetic (21\%), and 13 as having cryptogenic, now referred to as unknown (16\%). Twelve cases had a diagnosed epilepsy syndrome; 7 had Dravet syndrome, 5 had benign epilepsy with centrotemporal spikes (BECTS) and 5 had either Lennox Gastaut or West Syndrome. 
Amongst the 73 children with information regarding co-morbidities, 33 were classed as having developmental delay and/or intellectual impairment (45\%), six had cerebral palsy (8\%), five had a clinically diagnosed intercurrent pneumonia (7\%), and 26 had no co-morbid conditions $(36 \%)$.

\subsection{Epilepsy treatment}

At the time of death, patients were on a range of different antiepileptic drugs (AEDs). The number of AEDs used for treating epilepsy was provided for 69 children. These children were on an average of 1.4 AEDs (SD 0.9, range 0-3). Specific AEDs were reported in only 51 patients, with sodium valproate being the most frequently used (18 cases, 35\%). The next most commonly used AEDs were phenobarbitone, carbamazepine and phenytoin. Four children were on no AEDs at time of death $(8 \%)$.

\subsection{Neuropathological findings}

Neuropathological findings on autopsy were reported in 30 cases. Chronic neuropathological lesions were present in 17 cases (57\%). Seven children had evidence of acute hypoxic changes in brain (23\%). An unexpected finding of perivascular lymphocytic cuffing was present in three cases (10\%) in one case series despite no history of infection or inflammatory disease. ${ }^{7}$ Pulmonary oedema was noted in all 8 patients who underwent a complete autopsy, and myocardial fibre hypertrophy was noted in one case.

\section{Discussion}


Childhood SUDEP is rarely discussed in the literature compared to adult SUDEP; nevertheless it is an important subject for child neurologists, especially given our current inability to accurately identify high-risk patients, beyond those with frequent GTCS. Each study reported a small numbers of cases, likely due to the rarity of pediatric SUDEP. The majority of studies identified were retrospective case series and case control studies which used hospital records of tertiary centers in high-income countries. These studies have an inherent selection bias, being more likely to include patients with higher seizure frequency, resistance to antiepileptic drug therapy, atypical features, and longer duration of epilepsy. Our review included only one population-based study, which avoids this selection bias.

Details of 108 cases of pediatric SUDEP were gathered into a case series. Most of these cases were extracted from non-comparative studies, while the remainder came from comparative studies with heterogeneous control populations, which prevented any higher analysis. It is impossible to draw definitive conclusions from this non-comparative data, however due to the paucity of data on pediatric SUDEP narrative analysis of this case series provides new and valuable insights into possible risk factors for SUDEP in childhood, and as such can guide further research.

\subsection{Risk factors for SUDEP}

\subsubsection{Demographics}

Analysis of the case demographics of SUDEP victims reveals a heterogeneous patient group encompassing all ages and genders. The age of onset of epilepsy was low for patients in our case series; most of the children started having seizures before the age of 5 years (average 3.1 
years). A large Swedish case-control study of 6,880 adult SUDEP patients replicated this finding, finding that the relative risk of SUDEP was 7.7 times higher in patients with early onset epilepsy (0-15 years) than in patients with late onset (after 45 years). ${ }^{40}$

\subsubsection{Developmental delay/intellectual impairment}

Almost half of the children in the case series (45\%) had cognitive or developmental delay. Intellectual impairment and developmental delay are associated with medically refractory epilepsy and a greater risk of early all-cause mortality. ${ }^{41}$ Severe cognitive impairment has been associated with increased mortality in a study of epilepsy patients with over 40 years follow up. ${ }^{46}$ However, on multivariate analysis of this data the absence of 5-year terminal remission was the only significant risk factor for death. Conversely, in a prospective 10-year pediatric cohort study including 53 deaths (11 of which were SUDEP) moderate or severe intellectual impairment was significantly more frequent in patients who died from causes other than SUDEP. ${ }^{42}$ Interestingly, $33 \%$ of the children in this systematic review were reported to have no co-morbidities, suggesting that SUDEP can occur in children with uncomplicated epilepsy.

\subsubsection{Autopsy findings}

As the autopsies were performed in different studies across multiple institutions with possible variability in classification, the review is limited by the degree of detail of neuropathological studies. There was evidence of acute hypoxic and inflammatory changes in $23 \%$ of cases, with perivascular lymphocytic cuffing present in three cases. None of these patients had evidence of infection clinically prior to death. In addition, chronic lesions were present in the 
majority (61\%) of SUDEP victims. These findings do not necessarily reveal any risk factors for SUDEP as all pathological changes identified could be related to final common terminal events prior to death.

\subsubsection{Medications}

Our study does not have sufficient data to examine the relationship between specific AEDs and mortality. Sodium valproate was the most commonly used AED in the case series (35\%) followed by carbamazepine (25\%), phenobarbitone $(25 \%)$ and lamotrigine $(11 \%)$. The association between specific AEDs and the risk of SUDEP is complex and there have been contradictory reports in the adult literature. As the risk of SUDEP seems to correlate strongly with increased frequency of GTCS, the assumption that effective drug treatment would reduce its incidence seems reasonable. Poor compliance with AED treatment has been linked to increased risk of adult SUDEP. ${ }^{43}$ Although early reports implicated the sodium-channel blocking agent lamotrigine with an increased risk of SUDEP ${ }^{18,44}$ a recent meta-analysis found no relationship between any specific drugs and SUDEP. ${ }^{45}$

\subsubsection{Etiology of epilepsy}

Thirty-three per cent of the children in our case series had symptomatic epilepsy, encompassing structural, metabolic and immune causes. Idiopathic epilepsy (or genetic epilepsy as per ILAE Revised Terminology ${ }^{21}$ ), which is most common in the general population, was diagnosed in just $21 \%$ of children in our case series. The association between SUDEP and symptomatic epilepsy in our case series could be attributed to selection bias, however the association has been more reliably identified in the adult literature, primarily for epilepsy resulting from traumatic brain injury or encephalitis/meningitis. ${ }^{46}$ Several pediatric 
cohorts have also identified the association - analysis of four cohorts of children with newly diagnosed epilepsy found those whose disease was associated with neurodisability or an underlying brain condition had a SUDEP rate 2-3 times higher than other patients. ${ }^{23} \mathrm{~A}$ further prospective cohort study found children with symptomatic epilepsy had a standardized mortality ratio of 31.6 [95\% CI 18.4-50.6], versus 0.8 [95\% CI $0.02-4.2$ ] for idiopathic epilepsy. ${ }^{39}$ In contrast, a Dutch cohort of epilepsy patients in a tertiary neurology center showed no correlation between SUDEP and type of epilepsy for the 55 SUDEP cases included over a 5 -year period. ${ }^{37}$

In addition, $7 \%$ of the children in our series had Dravet syndrome, an SCN1A mutation related infantile epilepsy syndrome. This is the most well-described pediatric genetic epilepsy syndrome with increased SUDEP risk ${ }^{28}$, and the channelopathy resulting from SCN1A mutation may confer an increased risk of sudden cardiac death, compared to other epilepsy causes.

\subsubsection{Seizure type}

The majority of patients (88\%) in our review had GTCS, supporting previous studies that have found GTCS to be a major risk factor for both adults and children. ${ }^{5,11,42}$ Increased seizure burden has been previously associated with a higher SUDEP risk; a case control study reported that people who were not seizure free had a 23 -fold increased risk of SUDEP compared to people with fully controlled seizures. ${ }^{38}$ Other population-based studies found that adult SUDEP was associated with high seizure frequency (especially $>50$ seizures/year), multiple AEDs and early onset epilepsy. ${ }^{9}$ In this review children had on average 16.3 seizures per month. 


\subsection{Future directions}

Recently, there has been an increased interest in the molecular mechanisms underlying SUDEP, driven by whole exome sequencing (WES) studies. Mutations in ion channels expressed in the brain and the heart have been shown in animal and clinical case studies. ${ }^{21,47}$ WES data from SUDEP and epilepsy control patients demonstrated a significantly increased genome-wide polygenic burden per individual in the SUDEP group but no one single gene emerged as common to all cases. ${ }^{26}$

The North American SUDEP Registry in the United States and Canada has been set up for prospective surveillance of SUDEP cases and currently acts as a repository for clinical, imaging, tissue, genetic and physiological data. ${ }^{48}$ A similar SUDEP registry in the United Kingdom with a pediatric focus would provide a more population based approach for establishing incidence and risk factors of SUDEP in the UK. We thus propose a multidisciplinary approach to allow collection of high quality clinical information, tissue and DNA samples from UK epilepsy related mortality cases as possible to help in our search for understanding the mechanisms that lead to SUDEP.

\section{$\underline{\text { 5. Conclusions }}$}

In summary, from the limited evidence available it is apparent that a constellation of features associated with severe epilepsy such as early age of onset, high seizure frequency, intellectual impairment and developmental delay, multiple AED therapy, and structural abnormalities are associated with a higher risk of SUDEP. ${ }^{5,32,49,50}$ Although many of these are not necessarily modifiable risk factors, knowledge that they are associated with SUDEP can nonetheless be valuable for clinicians when counselling parents and families. 
There is a clear need for multiple large-scale, prospective, international community-based studies of both adult and child SUDEP to explore its pathogenesis, underlying risk factors and possible preventative measures - current single center studies have not identified sufficient numbers of SUDEP cases. Inconsistent and inaccurate death certification, the lack of agreed definitions, and use of different terminologies have historically hampered research into mortality in epilepsy. ${ }^{16}$ This has led to a probable under-appreciation of the public health burden of SUDEP and there is a strong need to raise awareness of SUDEP. This implies that it is not just clinicians who need to talk more about SUDEP, but also health policy makers, researchers and the public health community.

\section{$\underline{\text { Acknowledgments }}$}

We would like to thank all corresponding authors of studies included for providing details on their published pediatric SUDEP cases needed for this systematic review.

\section{$\underline{\text { Authors' contributions }}$}

OA conceptualized and designed the systematic review, drafted the initial manuscript, carried out the initial analyses and approved the final manuscript as submitted. HT assisted with designing the systematic review and made a substantial contribution to the manuscript, carried out the initial analyses, and approved the final manuscript as submitted. ED and AS conceptualized the systematic review, supervised the team, critically reviewed the manuscript and approved the final manuscript as submitted. All authors approved the final manuscript as submitted and agree to be accountable for all aspects of the work.

\section{Funding}


There is no funding associated with this work.

\section{$\underline{\text { Declarations of interest }}$}

We declare no competing interests. We confirm that we have read the Journal's position on issues involved in ethical publication and affirm that this report is consistent with those guidelines.

\section{$\underline{\text { References }}$}

1. Appleton R. Sudden unexpected death in epilepsy in children. Seizure 1997;6:175-177.

2. Nashef L. Sudden unexpected death in epilepsy: terminology and definitions. Epilepsia 1997; 38(Suppl 11): S6-8.

3. Nashef L, So E, Ryvlin P et al. Unifying the definitions of sudden unexpected death in epilepsy. Epilepsia 2012 1; 53: 227-233.

4. Annegers J, Coan S, Hauser W et al. Epilepsy, vagal nerve stimulation by the NCP system, all-cause mortality, and sudden, unexpected, unexplained death. Epilepsia 2000; 41: $549-553$.

5. Ficker D, So E, Annegers $\mathrm{J}$ et al. Population-based study of the incidence of sudden unexplained death in epilepsy. Neurology 1998; 51: 1270-1274.

6. Harden C, Tomson T, Gloss D et al. Practice guideline summary: sudden unexpected death in epilepsy incidence rates and risk factors Report of the Guideline Development, Dissemination, and Implementation Subcommittee of the American Academy of Neurology and the American Epilepsy Society. Neurology 2017; 88: 1674-80

7. Berg A, Nickels K, Wirrell E et al. Mortality risks in new-onset childhood epilepsy. Pediatrics 2013; 132: 124-131.

8. Appleton R. Mortality in paediatric epilepsy. Arch Dis Child 2003; 88: 1091-1094 
9. Timmings P. Sudden unexpected death in epilepsy: a local audit. Seizure 1993; 2: 287290.

10. Jick S, Cole T, Mesher R et al. Sudden unexpected death in young persons with primary epilepsy. Pharmacoepidemiol. Drug Saf 1992; 1: 59-64.

11. Nilsson L, Bergman U, Diwan V et al. Antiepileptic drug therapy and its management in sudden unexpected death in epilepsy: a case-control study. Epilepsia 2001; 42: 667-673.

12. Walczak T, Leppik I, D'Amelio $\mathrm{M}$ et al. Incidence and risk factors in sudden unexpected death in epilepsy: a prospective cohort study. Neurology 2001; 56: 519-525

13. Langan Y, Nashef L, Sander JW. Case-control study of SUDEP. Neurology 2005; 64: $1131-1133$

14. Ficker D. Sudden unexplained death and injury in epilepsy. Epilepsia 2000; 41: S7-12.

15. Ryvlin P, Nashef L, Lhatoo SD et al. Incidence and mechanisms of cardiorespiratory arrests in epilepsy monitoring units (MORTEMUS): a retrospective study. Lancet Neurol 2013; 12: 966-977.

16. Lhatoo S, Johnson A, Goodridge D et al. Mortality in epilepsy in the first 11 to 14 years after diagnosis: Multivariate analysis of a long-term, prospective, population-based cohort. Ann Neurol 2001; 49: 336-44

17. Ramachandran Nair R, Jack SM, Meaney BF et al. Sudden unexpected death in epilepsy: a search for risk factors. Epilepsy Behav 2007; 10: 138-141.

18. Ackers R, Besag F, Hughes E et al. Mortality Rates and Causes of Death in Children with Epilepsy Prescribed Antiepileptic Drugs. Drug safety 2011; 34: 403-413.

19. Aurlien D, Larsen J, Gjerstad L et al. Increased risk of sudden unexpected death in epilepsy in females using lamotrigine: A nested, case-control study. Epilepsia 2012; 53: 258-266. 
20. Bagnall R, Crompton D, Cutmore C et al. Genetic analysis of PHOX2B in sudden unexpected death in epilepsy cases. Neurology 2014; 83: 1018-1021

21. Berg A, Scheffer I. New concepts in classification of the epilepsies: entering the $21 \mathrm{st}$ century. Epilepsia 2011; 52: 1058-1062.

22. Donner EJ, Smith CR, Snead OC. Sudden unexplained death in children with epilepsy. Neurology. 2001; 57: 430-434.

23. Doumlele K, Friedman D, Buchhalter J, Donner EJ, Louik J, Devinsky O. Sudden unexpected death in epilepsy among patients with benign childhood epilepsy with centrotemporal spikes. JAMA Neurol 2017; 74: 645-649

24. Geerts A, Arts WF, Stroink H et al. Course and outcome of childhood epilepsy: A 15-year follow-up of the Dutch Study of Epilepsy in Childhood. Epilepsia 2010; 51: 1189-1197.

25. Kinney H, McDonald A, Minter M et al. Witnessed sleep-related seizure and sudden unexpected death in infancy: a case report. Forensic Sci Med Pathol 2013; 9: 418-421

26. Klassen T, Bomben V, Patel A et al. High-resolution molecular genomic autopsy reveals complex sudden unexpected death in epilepsy risk profile. Epilepsia 2014; 55: e6-12.

27. Labate A, Tarantino P, Palamara G et al. Mutations in PRRT2 result in familial infantile seizures with heterogeneous phenotypes including febrile convulsions and probable SUDEP. Epilepsy Res 2013; 104: 280-284.

28. Langan Y, Nashef L, Sander JW. Sudden unexpected death in epilepsy: a series of witnessed deaths. J Neurol Neurosurg Psychiatry 2000; 68: 211-213.

29. Le Gal F, Korff C, Monso-Hinard C et al. A case of SUDEP in a patient with Dravet syndrome with SCN1A mutation. Epilepsia 2010; 51: 1915-1918.

30. Lear-Kaul K, Coughlin L, Dobersen M. Sudden unexpected death in epilepsy: a retrospective study. J Neurol Neurosurg Psychiatry 2005; 26 11-17.

31. Leu C, Balestrini S, Maher B et al. Genome-wide polygenic burden of rare deleterious 
variants in sudden unexpected death in epilepsy. EBioMedicine 2015; 2: 1063-1070.

32. McGregor A, Wheless J. Pediatric experience with sudden unexplained death in epilepsy at a tertiary epilepsy center. J. Child. Neurol 2006; 21:782-787.

33. Nashef L, Fish D, Garner S et al. Sudden death in epilepsy: a study of incidence in a young cohort with epilepsy and learning difficulty. Epilepsia 1995; 36: 1187-1194.

34. Sillanpää M, Shinnar S. Long-term mortality in childhood-onset epilepsy. $N$ Engl J Med 2010; 363: 2522-2529.

35. Surges R, Adjei P, Kallis C et al. Pathologic cardiac repolarization in pharmacoresistant epilepsy and its potential role in sudden unexpected death in epilepsy: a case-control study. Epilepsia 2010; 51: 233-242.

36. Swallow R, Hillier C, Smith P. Sudden unexplained death in epilepsy (SUDEP) following previous seizure-related pulmonary oedema: case report and review of possible preventative treatment. Seizure 2002; 11: 446-448.

37. Tu E, Bagnall R, Duflou J et al. Post-Mortem Review and Genetic Analysis of Sudden Unexpected Death in Epilepsy (SUDEP) Cases. Brain Pathol 2011; 21: 201-208.

38. Vlooswijk M, Majoie H, De Krom M et al. SUDEP in the Netherlands: a retrospective study in a tertiary referral center. Seizure 2007; 16: 153-159.

39. Weber P, Bubl R, Blauenstein U et al. Sudden unexplained death in children with epilepsy: A cohort study with an eighteen-year follow-up. Acta Paediatr 2005; 94: 564567.

40. Nilsson L, Farahmand BY, Persson PG et al. Risk factors for sudden unexpected death in epilepsy: a case-control study. Lancet 1999; 353: 888-893.

41. Shinnar S, Pellock JM. Update on the epidemiology and prognosis of pediatric epilepsy. $J$ Child Neurol 2002; 17(Suppl 1): S4-S17. 
42. Terra V, Scorza F, Arida R et al. Mortality in children with severe epilepsy: 10 years of follow-up. Arq Neuropsiquiatr 2011; 69: 766-769.

43. T.llez-Zenteno JF, Ronquillo LH, Wiebe S. Sudden unexpected death in epilepsy: evidence-based analysis of incidence and risk factors. Epilepsy Res 2005; 65: 101-115.

44. Nashef L, Garner S, Sander J et al. Circumstances of death in sudden death in epilepsy: interviews of bereaved relatives. J Neurol Neurosurg Psychiatry 1998; 64: 349-352.

45. Hesdorffer D, Tomson T, Benn E et al. Combined analysis of risk factors for SUDEP. Epilepsia 2011; 52: 1150-1159.

46. Johnson J, Hofman N, Haglund $\mathrm{C}$ et al. Identification of a possible pathogenic link between congenital long QT syndrome and epilepsy. Neurology 2009; 72: 224-231.

47. Annegers, S.P. Coan, SUDEP: overview of definitions and review of incidence data, Seizure 1999; 8: 347-352.

48. Donner E, Devinsky O. Registries for SUDEP research. In: Hanna J, Panelli R, Jeffs T, Chapman D, editors. Continuing the global conversation [online]. SUDEP Action, SUDEP Aware \& Epilepsy Australia; 2014 [retrieved 05/09/16]. Available from: Www.sudepglobalconversation.com.

49. Nickels K, Grossardt B, Wirrell E. Epilepsy-related mortality is low in children: A 30year population-based study in Olmsted County, MN. Epilepsia 2012; 53: 2164-2171.

50. Pedley T, Hauser W. Sudden death in epilepsy: a wake-up call for management. Lancet 2002; 359: 1790-1791 


\section{$\underline{\text { Figure and table legends }}$}

Table 1: Description of 22 studies included in systematic review

Figure 1: Sub-classification of SUDEP (Modified from Nashef et al 2012) ${ }^{3}$

Figure 2: Systematic Review PRISMA Flow Diagram

Figure 3: Tables of characteristics of 108 childhood SUDEP cases from 22 studies in systematic review 
